\title{
Evaluación de sistemas térmicos y fotovoltaicos solares en unidades de producción agropecuaria, Región Huetar Norte, Costa Rica ${ }^{1}$
}

\section{Evaluation of thermal and photovoltaic solar systems in agricultural production units, Northern Huetar Region, Costa Rica}

\author{
Tomás de Jesús Guzmán-Hernández ${ }^{2}$,Freddy Araya-Rodríguez², Javier Mauricio Obando-Ulloa ${ }^{2}$, \\ Mikel Rivero-Marcos ${ }^{2}$, Guillermo Castro-Badilla ${ }^{3}$
}

\begin{abstract}
Resumen
La dependencia por los combustibles fósiles insta a buscar alternativas de producción más limpias que contribuyan a mitigar los efectos del cambio climático. El objetivo del presente trabajo fue determinar el potencial del uso de la energía solar para el calentamiento de agua y generación de energía eléctrica. El estudio se desarrolló en la lechería de la Sede Regional San Carlos, Instituto Tecnológico de Costa Rica, entre mayo de 2015 y abril de 2016. Se determinó la cantidad de electricidad producida y las temperaturas alcanzadas, por medio de los sistemas fotovoltaicos y térmicos instalados mediante un registro computarizado. También se calculó la cantidad de dióxido de carbono equivalente que se dejó de emitir a la atmósfera y, el ahorro energético y económico de su consumo. El uso de los sistemas de energía solar permitió lograr un autoabastecimiento de energía eléctrica entre 30 y $40 \%$ del consumo total de la unidad productiva. En la producción de energía para calentar el agua para la limpieza y sanitización de los equipos de ordeño, el sistema térmico fue capaz de lograr un incremento entre 20 y $37{ }^{\circ} \mathrm{C}$, lo que representó más del $70 \%$ de la energía necesaria para alcanzar la temperatura requerida $(70$ ํㅡ y un ahorro económico de aproximadamente $\$ 90$ mensuales. Estos sistemas contribuyeron a disminuir la dependencia de los combustibles fósiles, al mejorar la eficiencia económica y productiva en unidades de producción agropecuaria en la Región Huetar Norte de Costa Rica.
\end{abstract}

Palabras clave: energía eléctrica, energía solar, producción agropecuaria, sistema fotovoltaico, sistema térmico.

\begin{abstract}
The dependence on fossil fuels urges society to seek for clean energy alternatives, in order to mitigate the effects of climate change. The objective of this study was to determine the potential of solar energy used for water heating and electricity generation. The study was conducted at the dairy of the Technology Institute of Costa Rica, San Carlos Headquarter, from May 15 to April 2016. The data related to the amount of the electricity produced and the temperature reached by water was obtained from the installed photovoltaic and thermal systems, the data was
\end{abstract}

\footnotetext{
1 Recibido: 3 de octubre, 2016. Aceptado: 8 de febrero, 2017. Formó parte de un proyecto de investigación del Instituto Tecnológico de Costa Rica (ITCR), Sede Regional San Carlos; financiado con fondos del Programa de Regionalización del Consejo Nacional de Rectores (CONARE), Ministerio de Agricultura y Ganadería (MAG) y Vicerrectoría de Investigación y Extensión del ITCR, Costa Rica.

2 Instituto Tecnológico de Costa Rica, Programa de Doctorado en Ciencias Naturales para el Desarrollo (DOCINADE), Sede Regional San Carlos. Apartado postal 223-21001 Ciudad Quesada, Alajuela, Costa Rica. Teléfono: (506) 2401-3020. Fax: (506) 2475-5395. tjguzman@ itcr.ac.cr; faraya@itcr.ac.cr; jaobando@itcr.ac.cr; mriveromarcos@gmail.com

3 Instituto Tecnológico de Costa Rica, Escuela de Electrónica, Sede Regional San Carlos, Costa Rica. gucastro@itcr.ac.cr
} 
recorded by a computerized register. The obtained information about electricity production allowed researchers to calculate the amount of carbon dioxide equivalent that was not emitted into the atmosphere, and also the acquired economic saving on consumption. The use of these systems allowed the production unit have a self- sufficient source of electrical energy percentage, actually around 30 to $40 \%$ of the total electrical consumption. According to the energy production, the solar thermal system was capable to increase water temperature between 20 to $37^{\circ} \mathrm{C}$, temperature that represents more than $70 \%$ of the energy needed in order to reach the required water temperature $\left(70{ }^{\circ} \mathrm{C}\right)$ for cleaning and sanitizing the milking equipment, and also an economical saving around $\$ 90$ per month was achieved. The results showed that these systems allow to improve the economical and productive efficiency of agricultural production units in the Northern Huetar Region of Costa Rica.

Keywords: electrical energy, solar energy, agricultural production, photovoltaic system, thermal system.

\section{Introducción}

En los últimos años, la producción y consumo de energías renovables a nivel internacional ha adquirido un mayor auge como resultado del encarecimiento de los combustibles fósiles y sus derivados, los altos índices de emisiones contaminantes, así como la necesidad de impulsar el compromiso con el desarrollo sostenible (IglesiasFerrer y Morales-Salas, 2013). Es fundamental que el progreso general de la humanidad sea armónico con un desarrollo positivo y sostenible, entendiéndose como aquel que satisface las necesidades del presente, sin crear problemas y sin comprometer las demandas de las futuras generaciones (Lamela, 2005).

La ganadería es responsable del 18\% de las emisiones mundiales de gases de efecto invernadero (GEI), más aún que el transporte, de acuerdo con el informe presentado por la Organización de las Naciones Unidas para la Alimentación y la Agricultura (FAO) sobre el impacto ambiental de esta actividad (Matthews, 2006). Por lo tanto, urge aplicar tecnologías que minimicen este impacto generado en los procesos productivos del sector.

En el cantón de San Carlos (provincia de Alajuela, Costa Rica) se encuentra el 55\% de los asociados de la Cooperativa de Productores de Leche Dos Pinos R.L. (850 productores en total), quienes producen más del 50\% de la producción nacional (0,6 millones de kilogramos de leche). Entre los procesos productivos del sector ganadero y lechero que inciden negativamente en la huella de carbono local, se encuentra el consumo eléctrico de las plantas agroindustriales.

Del $100 \%$ de radiación solar, solo un $51 \%$ de esta llega a la superficie terrestre. Del resto, un $19 \%$ es absorbido por las nubes y el otro $30 \%$ se pierde hacia el espacio (Taiz y Zeiger, 2002; Espejo-Marín, 2004). Actualmente, para captar la energía solar que incide sobre la superficie se usan distintos tipos de sistemas térmicos solares:

- Colectores solares para el calentamiento del agua: pueden ser de circuito abierto o cerrado, y normalmente son para uso doméstico. En estos sistemas, el agua de consumo se traslada a través de un circuito abierto que pasa directamente por los colectores solares y, en el cerrado, el agua caliente se almacena en un depósito para su posterior uso. Este sistema reduce costos y es más eficiente, pero presenta problemas en zonas con temperaturas bajas, así como con alta concentración de sales que acaban obstruyendo el sistema. Además, en las instalaciones de circuito cerrado se distinguen dos tipos de flujo: por termosifón (en el que el agua circula por diferencia de densidad) y forzado (en el que la circulación del agua se hace mediante una bomba) (Prosperi y Croci, 2005; Khan et al., 2010; Quirós, 2011). Debido a que la radiación solar no es siempre suficiente para calentar agua durante todo el año, es recomendable conectar el sistema solar a un sistema convencional de calentamiento (Shyam, 2005). 
- Equipos o módulos fotovoltaicos: están formados por un conjunto de celdas que producen electricidad a partir de la radiación solar que incide sobre ellos, bajo condiciones estandarizadas de radiación $\left(1000 \mathrm{~W} / \mathrm{m}^{2}\right)$ y temperatura de la célula $\left(25^{\circ} \mathrm{C}\right)$. Los equipos fotovoltaicos se dividen en monocristalinos, compuestos de secciones de un único cristal de silicio; policristalinos, cuando están formados por pequeñas partículas cristalizadas; y amorfos, cuando el silicio no se ha cristalizado. Su efectividad es mayor cuanto mayor son los cristales, pero también su peso, grosor y costo. El rendimiento de los primeros puede alcanzar el $20 \%$ mientras que el de los últimas, aunque su peso y costo es muy inferior, no llega al 10\% (Espejo-Marín, 2004; González, 2009).

En el VI Plan Nacional de Energía 2012-2030 se indicó que Costa Rica presenta un potencial teórico solar de 10000 MW (MINAET, 2011); sin embargo, su grado de utilización es mínimo. Por esta razón, el Ministerio de Ambiente, Energía y Telecomunicaciones (MINAET) en la línea de acción de la estrategia 2.2 para el sector energía, insta a promover programas de ahorro energético en los macroconsumidores, por lo que, ha focalizado el estudio en las oportunidades y el potencial que tiene la Región Huetar Norte con fuentes de energía alternativas. En este punto, se ha optado por la tecnología solar como alternativa viable y efectiva, puesto que esta región es una de las mejores zonas de radiación solar en Costa Rica, de acuerdo con el Instituto Costarricense de Electricidad (ICE, 2015).

Debido a que el Instituto Tecnológico de Costa Rica (ITCR) tiene la misión de colaborar con el desarrollo del país, con diversos planes y programas de sensibilización y capacitación en sistemas de producción limpia, se diseñaron e implementaron dos sistemas solares para su evaluación técnica, económica y ambiental en la lechería de su Sede Regional San Carlos (ITCR-SSC).

El objetivo del presente trabajo fue determinar el potencial del uso de la energía solar para el calentamiento de agua y la generación de energía eléctrica.

\section{Materiales y métodos}

Se diseñó, construyó e instaló dos dispostivos solares fotovoltaicos e híbridos-termosifónicos en la lechería de la Sede Regional San Carlos del Instituto Tecnológico de Costa Rica en Santa Clara de San Carlos, Alajuela (ITCRSSC). Una vez que los sistemas estuvieron instalados y en funcionamiento, se evaluaron los resultados obtenidos desde mayo de 2015 a abril de 2016.

La temperatura ambiental media $\left({ }^{\circ} \mathrm{C}\right)$, precipitación mensual acumulada $(\mathrm{mm})$ y radiación solar $(\mathrm{kWh} /$ $\mathrm{m}^{2}$ ), registradas por la estación meteorológica del ITCR-SSC cada treinta minutos a lo largo de un año, fueron monitoreadas por el software climatológico WeatherLink 6.0.3 (Davis Instruments Corporation, Hayward, California, Estados Unidos).

\section{Sistemas fotovoltaicos}

Los sistemas fotovoltaicos instalados en la lechería del ITCR-SCC constaron de ocho colectores solares (Yingli solar, HIT-N235SE10, Baoding, China) de $235 \mathrm{Wp}$ y 1,26 $\mathrm{m}^{2}$ cada uno, provistos con un micro inversor para generar corriente alterna e introducirla directamente en la red eléctrica.

La energía eléctrica $(\mathrm{kWh})$ generada por los sistemas fotovoltaicos se registró cada 15 min a través de un equipo de transmisión de datos inalámbricos (Enphase Energy Inc., Petaluma, California, Estados Unidos).

La eficiencia de estos sistemas se calculó con base en la cantidad de energía eléctrica generada en función de la intensidad de radiación solar recibida $\left(\mathrm{kWh} / \mathrm{m}^{2}\right)$. 


\section{Sistemas termosifónicos}

Los sistemas termosifónicos instalados en la lechería del ITCR-SCC constaron de ocho colectores de placa plana con cubierta de poliuretano (Ecosun, HIT-N235SE10, Buenos Aires, Argentina) de 2,10 $\mathrm{m}^{2}$ cada uno, y con tubos de cobre en posición longitudinal para la circulación del agua. Cada cuatro colectores solares fueron conectados a un tanque acumulador primario con capacidad para 6501 de agua, desde cuya parte superior se canalizaba el agua caliente a un tanque acumulador secundario de 6501 de capacidad, el cual tenía acoplado un sistema auxiliar eléctrico y una válvula de escape en caso de vaporización del agua (Figura 1). El agua caliente asciende por diferencia de densidad hasta depositarse en el sistema de acumulación primaria. De su parte superior se canaliza a un segundo tanque que, junto con el sistema auxiliar, permite disponer de agua caliente a alta temperatura.

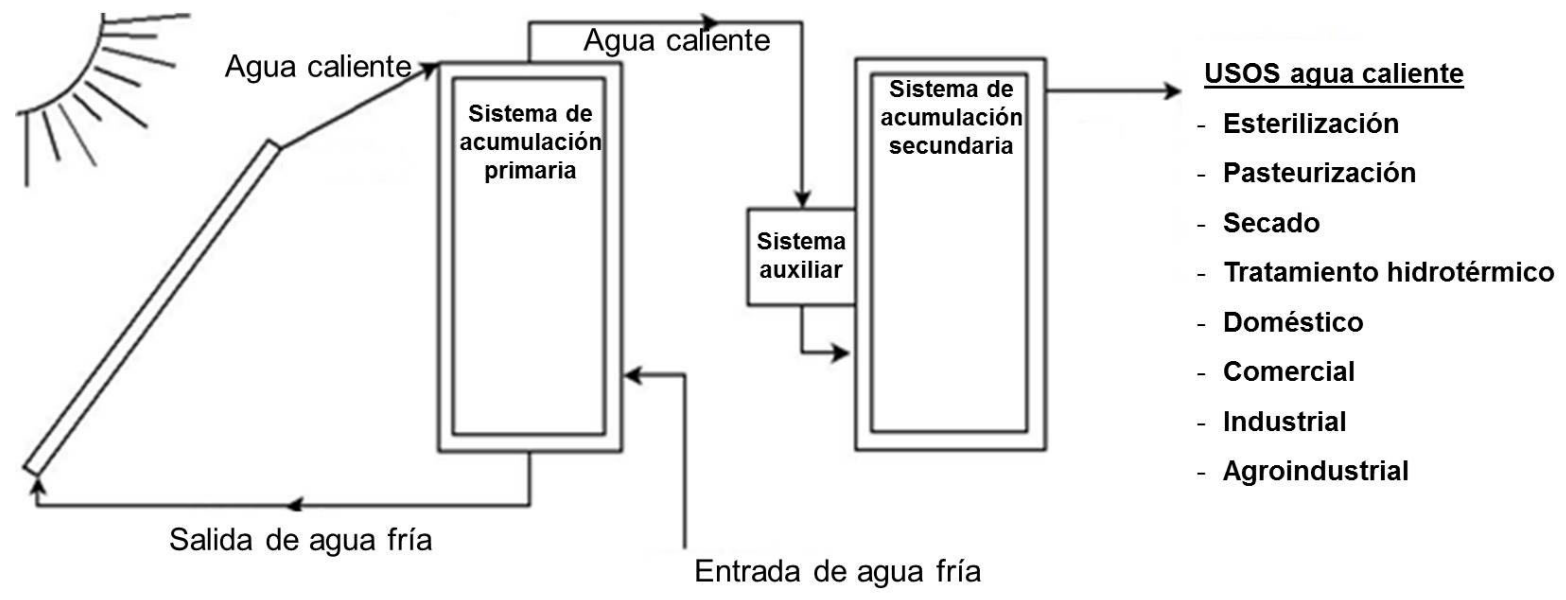

Figura 1. Esquema básico del sistema termosifónico híbrido instalado en la lechería del Instituto Tecnológico de Costa Rica, Sede Regional San Carlos (ITCR-SSC), Costa Rica. 2015.

Figure 1. Basic diagram of the hybrid-thermosiphon system installed at the dairy of the Technology Institute of Costa Rica, San Carlos Headquarter (ITCR-SSC), Costa Rica. 2015.

Las temperaturas alcanzadas $\left({ }^{\circ} \mathrm{C}\right.$ ) en el sistema termosifónico (temperatura de entrada del agua a los colectores, temperaturas del agua a la salida del tanque acumulador primario 1 y 2 , y temperatura a la entrada del tanque acumulador secundario) se determinaron con un termómetro y un sistema automatizado de registro de datos. Con base en la temperatura alcanzada por el agua tras el paso por los colectores, se calculó el ahorro energético que correspondió al porcentaje de energía suministrado por el sistema termosifónico híbrido para alcanzar la temperatura requerida del agua $\left(70{ }^{\circ} \mathrm{C}\right)$ para las operaciones de limpieza de la lechería y sanitización de los equipos de ordeño (ecuación 1).

\section{Ahorro energético $(\%)=\frac{\text { Temperatura agua salida del colector }}{\text { Temperatura requerida }} \times 100 \quad$ (ecuación 1)}

La energía equivalente al incremento de la temperatura se calculó con la ecuación 2 (transferencia de calor; Henley, 1973), estimando un consumo promedio de 2001 de agua al día. 
$Q(k W h)=V \times \varrho \times C p x \times \Delta T \times 2,7 \times 10^{-7} \mathrm{kWh} / \mathrm{J}$

(ecuación 2)

donde,

V: volumen del agua consumido al día $\left(0,2 \mathrm{~m}^{3} /\right.$ día $)$.

@: densidad del agua $\left(1000 \mathrm{~kg} / \mathrm{m}^{3}\right)$.

Cp: calor específico del agua $\left(4187 \mathrm{~J} / \mathrm{kg} \cdot{ }^{\circ} \mathrm{C}\right)$.

$\Delta \mathrm{T}$ : incremento de la temperatura del agua, generado por los colectores $\left({ }^{\circ} \mathrm{C}\right)$.

$2,7 \times 10^{-7}$ : factor de conversión de kilowatt hora $(\mathrm{kWh})$ a julio $(\mathrm{J})$.

El rendimiento del sistema se calculó por medio de los valores de radiación incidente, temperatura ambiente y la temperatura del agua a la entrada y a la salida del colector solar, según la ecuación 3 (ASRHAE, 2003).

$R=\eta_{0}-\left[U_{L}\left(T_{m}-T_{a}\right) / I\right]$

(ecuación 3)

donde,

$\eta_{0}$ : Rendimiento óptico, indica el porcentaje de los rayos solares que penetran la cubierta transparente del colector $(\%)$

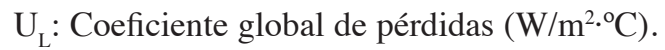

$\mathrm{T}_{\mathrm{m}}$ : temperatura media del agua a su paso por el colector $\left({ }^{\circ} \mathrm{C}\right)$.

$\mathrm{T}_{\mathrm{a}}$ : temperatura media del agua previamente a su entrada al colector $\left({ }^{\circ} \mathrm{C}\right)$.

I: radiación incidente total sobre el colector $\left(\mathrm{W} / \mathrm{m}^{2}\right)$.

En el Cuadro 1 se muestran los valores típicos de los parámetros característicos de eficiencia óptica $\left(\eta_{0}\right)$, coeficiente global de pérdidas $\left(\mathrm{U}_{\mathrm{L}}\right)$ y el rango normal de temperaturas de trabajo para distintos tipos de colectores. En este estudio, el rendimiento diario de los sistemas térmicos solares se calculó en intervalos de 30 min, desde las 5:30 hasta las $18 \mathrm{~h}$. Los sistemas térmicos instalados en la lechería del ITCR-SSC eran de cubierta simple con un rango de temperatura de 10 a $60{ }^{\circ} \mathrm{C}$, por lo que los valores orientativos que se tomaron como factor de conversión y de pérdidas térmicas fueron 0,8 y 7 , respectivamente.

Cuadro 1. Valores de los parámetros para calcular el rendimiento de los colectores térmicos. Lechería del Instituto Tecnológico de Costa Rica, Sede Regional San Carlos (ITCR-SSC), Costa Rica. 2016.

Table 1. Parameter values used to calculate the performance of the thermal collectors. Cambiar por Technology Institute of Costa Rica's Dairy, San Carlos Headquarter (ITCR-SSC), Costa Rica. 2016.

\begin{tabular}{lccc}
\hline Tipo de colector & Factor de conversión $\left(\boldsymbol{\eta}_{\mathbf{0}}\right)$ & $\begin{array}{c}\text { Factor de pérdidas térmicas } \\
\mathbf{U}_{\mathbf{L}}\left(\mathbf{W} / \mathbf{m}^{\mathbf{2} \cdot{ }^{\circ}} \mathbf{C}\right)\end{array}$ & Rango de temperatura $\left({ }^{\mathbf{0}} \mathbf{C}\right)$ \\
\hline Sin cubierta & 0,9 & $15-25$ & $10-40$ \\
Cubierta simple & 0,8 & 7 & $10-60$ \\
Cubierta doble & 0,65 & 5 & $10-80$ \\
Superficie selectiva & 0,8 & 5 & $10-80$ \\
Tubos de vacío & 0,7 & 2 & $10-130$ \\
\hline
\end{tabular}

Fuente/Source: Díaz, 2015. 
El impacto ambiental de estos sistemas se determinó por la relación de los $\mathrm{kg}$ de $\mathrm{CO}_{2}$ equivalentes que no se emiten a la atmósfera como consecuencia de la energía generada $(\mathrm{kWh})$ por los sistemas para uso eléctrico. De acuerdo con el Instituto Meteorológico Nacional (IMN, 2016) en Costa Rica, un KWh equivale a 0,0381 kg $\mathrm{CO}_{2}$.

El ahorro económico de los sistemas solares se calculó con base en la producción energética durante el tiempo que han funcionado en la unidad de estudio. De acuerdo con la tarifa vigente de la Cooperativa de Electrificación Rural de San Carlos R.L. (COOPELESCA R.L.), para un consumo mensual superior a los $3000 \mathrm{kWh}$ (como es el caso de las lecherías), el cargo por energía se sitúa en los US\$1,34. A este monto se le debe sumar US\$2 200,69 por $\mathrm{kW}$ de potencia instalada (los sistemas fotovoltaicos instalados en la lechería del ITCR-SSC tienen una potencia de $2 \mathrm{~kW}$ ), US $\$ 0,60$ por $\mathrm{kWh}$ de energía consumida en gastos de alumbrado público y un $5 \%$ del monto total por la energía eléctrica consumida, a partir de consumos mayores a los $250 \mathrm{kWh}$ mensuales (ICE, 2015).

Finalmente, el tiempo de recuperación de la inversión inicial (\$10.000 USD en total) se estimó teniendo en cuenta el ahorro económico del año evaluado (ecuación 4).

$$
\text { Recuperación de la inversión (años) }=\frac{\text { Costo inicial }}{\text { Ahorro económico anual }} \quad \text { (ecuación 4) }
$$

\section{Resultados}

\section{Sistemas fotovoltaicos}

En Santa Clara de San Carlos se registró una temperatura promedio de $26^{\circ} \mathrm{C}$ y una radiación mensual de 115 $\mathrm{kWh} / \mathrm{m}^{2}$ durante el período en estudio (mayo 2015 a abril de 2016; Figura 2).

De acuerdo con estas condiciones, el sistema fotovoltaico generó más de $200 \mathrm{~kW} / \mathrm{h}$ en los meses de agosto y diciembre de 2015, respectivamente. Este volumen de producción energética se mantuvo hasta abril de 2016,

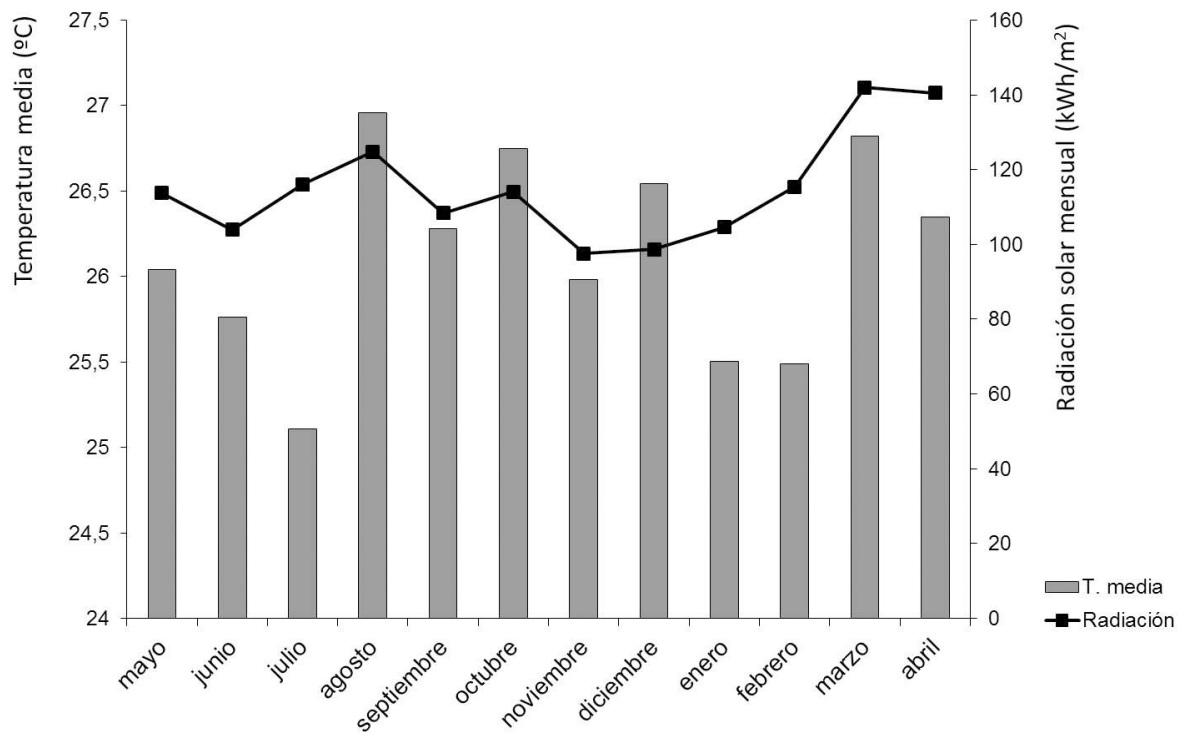

Figura 2. Temperatura media y radiación mensual registradas desde mayo 2015 a abril 2016 en Santa Clara de San Carlos, Costa Rica. Figure 2. Mean temperature and monthly radiation recorded from May 2015 to April 2016 in Santa Clara, San Carlos, Costa Rica. 
logrando una producción máxima (270 KwH) en marzo de 2016 (Figura 3), la cual a su vez presentó una correlación alta $\left(r^{2}=0,63\right)$ con la radiación solar que se aprecia notablemente a lo largo de un día cuando la radiación solar y la producción eléctrica de los sistemas fotovoltaicos es máxima al mediodía (aprox. $800 \mathrm{Wh} / \mathrm{m}^{2}$ y $100 \mathrm{Wh} / \mathrm{m}^{2}$, respectivamente; Figura 4).

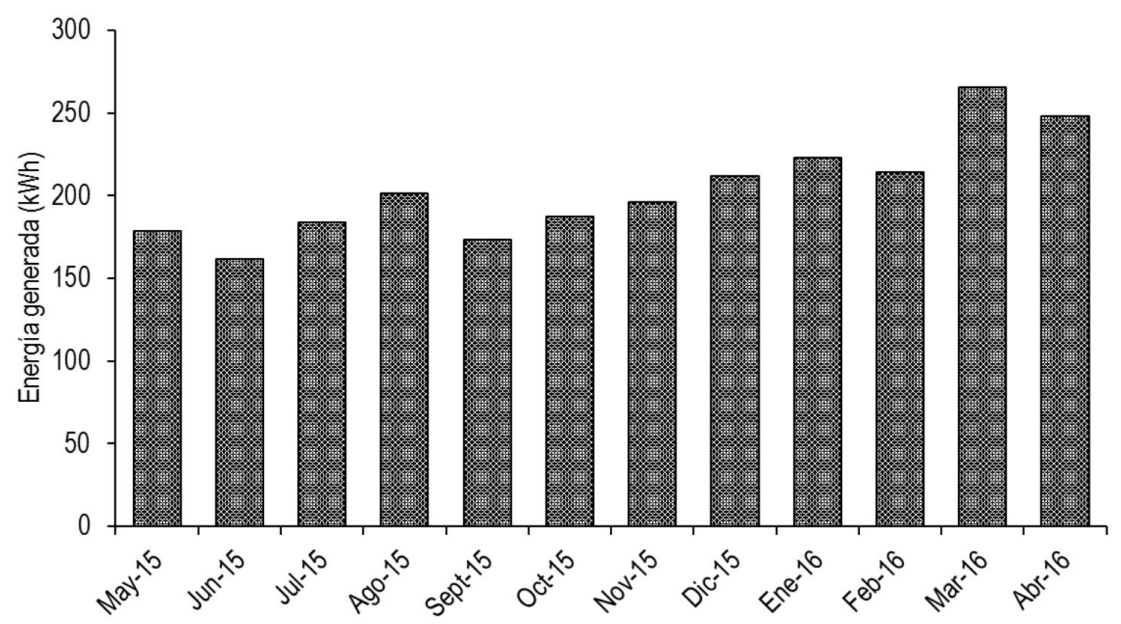

Figura 3. Energía generada por los sistemas fotovoltaicos de mayo 2015 a abril de 2016 en la lechería del Instituto Tecnológico de Costa Rica, Sede Regional San Carlos (ITCR-SSC). Costa Rica.

Figure 3. Energy generated by the photovoltaic systems from May 2015 to April 2016 in the dairy of the Technology Institute of Costa Rica, San Carlos Headquarter (ITCR-SSC). Costa Rica.

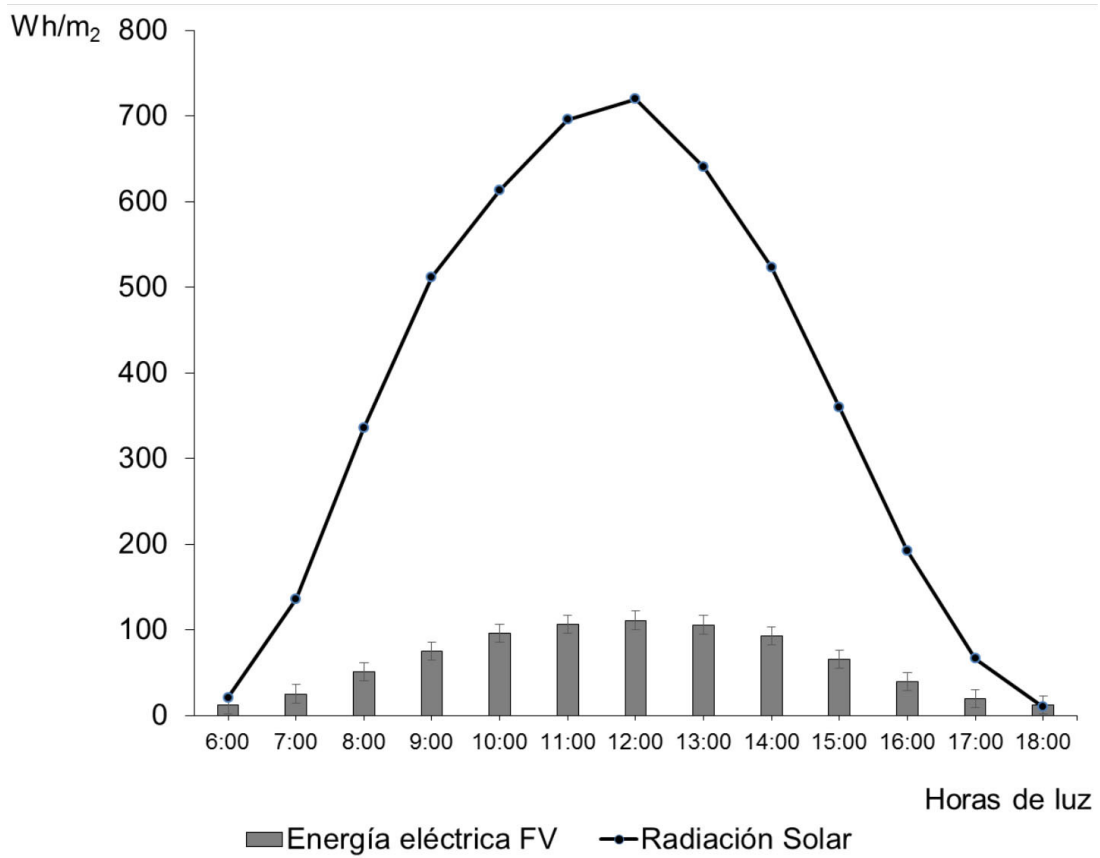

Figura 4. Promedio diario de la radiación solar y energía eléctrica fotovoltaica generada desde mayo 2015 a abril 2016. Lechería del Instituto Tecnológico de Costa Rica, Sede Regional San Carlos (ITCR-SSC), Costa Rica.

Figure 4. Daily mean of solar radiation and photovoltaic electrical energy generated from May 2015 to April 2016. Technology Institute of Costa Rica's Dairy, San Carlos Headquarter (ITCR-SSC), Costa Rica. 
El rendimiento de los sistemas fotovoltaicos en el período analizado presentó una tendencia entre $15 \%$ y $21 \%$, cuyo máximo valor se presentó en diciembre 2015 y enero 2016 (Cuadro 2).

Cuadro 2. Rendimiento de los sistemas fotovoltaicos en función de la energía generada a partir de la radiación incidente, mayo 2015 a abril de 2016. Lechería del Instituto Tecnológico de Costa Rica, Sede Regional San Carlos (ITCR-SSC).

Table 2. Performance of photovoltaic systems based on the energy generated from the incident radiation. May 2015 to April 2016. Technology Institute of Costa Rica's Dairy, San Carlos Headquarter (ITCR-SSC).

\begin{tabular}{lccc}
\hline Meses & Energía generada $\left(\mathbf{k W h} / \mathbf{m}^{2} \cdot \mathbf{m e s}\right)$ & Energía solar incidente $\left(\mathbf{k W h} / \mathbf{m}^{2} \cdot \mathbf{m e s}\right)$ & Rendimiento $\mathbf{( \% )}$ \\
\hline may-15 & 17,73 & 113,73 & 15,59 \\
jun-15 & 16,01 & 103,94 & 15,40 \\
jul-15 & 18,22 & 116,05 & 15,70 \\
ago-15 & 19,94 & 124,76 & 15,99 \\
sep-15 & 17,19 & 108,43 & 15,86 \\
oct-15 & 18,59 & 114,05 & 16,30 \\
nov-15 & 19,46 & 97,53 & 19,95 \\
dic-15 & 21,00 & 98,67 & 21,28 \\
ene-16 & 22,11 & 104,55 & 21,15 \\
feb-16 & 115,38 & 18,40 \\
mar-16 & 21,23 & 141,94 & 18,57 \\
abr-16 & 26,35 & 140,50 & 17,49 \\
\hline
\end{tabular}

Durante el período analizado, los sistemas fotovoltaicos generaron un total de $136,1 \mathrm{~kg}$ equivalentes de $\mathrm{CO}_{2}$ (Cuadro 3).

Cuadro 3. Kilogramos de $\mathrm{CO}_{2}$ equivalente a la energía generada por los sistemas fotovoltaicos. Lechería del Instituto Tecnológico de Costa Rica, Sede Regional San Carlos (ITCR-SSC), mayo 2015 a abril de 2016, Costa Rica.

Table 3. Kilograms of $\mathrm{CO}_{2}$ equivalent to the energy generated by the photovoltaic systems. Technology Institute of Costa Rica's Dairy, San Carlos Headquarter (ITCR-SSC), May 2015 to April 2016, Costa Rica.

\begin{tabular}{ccc}
\hline Mes & Energía generada por los sistemas fotovoltaicos $\mathbf{( k W h )}$ & Kg equivalente de $\mathbf{C O}_{2}$ \\
\hline may-15 & 178,76 & 10,0 \\
jun-15 & 161,34 & 9,0 \\
jul-15 & 183,69 & 10,2 \\
ago-15 & 201,0 & 11,2 \\
sep-15 & 173,3 & 9,7 \\
oct-15 & 187,4 & 10,4 \\
nov-15 & 196,1 & 10,9 \\
dic-15 & 211,6 & 11,8 \\
ene-16 & 222,9 & 12,4 \\
feb-16 & 214,0 & 11,9 \\
mar-16 & 265,7 & 14,8 \\
abr-16 & 248,0 & 13,8 \\
\hline Total anual & 2443,79 & 136,1 \\
\hline
\end{tabular}




\section{Sistema termosifónico}

El sistema solar termosifónico alcanzó una temperatura promedio de aproximadamente $48,5^{\circ} \mathrm{C}$ y generó alrededor de 140,34 kWh mensualmente, lo que permitió ahorrar un 74\% de la energía necesaria para alcanzar la temperatura requerida del agua para las operaciones de limpieza y desinfección de la lechería del ITCR-SSC (Cuadro 4).

Cuadro 4. Energía generada mensualmente por los sistemas solares termosifónicos instalados en la lechería del Instituto Tecnológico de Costa Rica, Sede Regional San Carlos (ITCR-SSC). Costa Rica. Mayo 2015 a Abril 2016.

Table 4. Monthly energy generated by the thermosyphonic solar systems installed at the Technology Institute of Costa Rica's Dairy, San Carlos Headquarter (ITCR-SSC). May 2015 to April 2016.

\begin{tabular}{|c|c|c|c|c|c|c|}
\hline \multirow[b]{2}{*}{ Mes } & \multicolumn{4}{|c|}{ Temperatura del agua $\left({ }^{\circ} \mathrm{C}\right)$} & \multirow{2}{*}{$\begin{array}{c}\text { Ahorro } \\
\text { energético (\%) }\end{array}$} & \multirow{2}{*}{$\begin{array}{c}\text { Energía aportada } \\
\text { por el sistema }(\mathrm{kWh} / \\
\text { mes) }\end{array}$} \\
\hline & Inicial $\left(\mathbf{T}_{\mathrm{i}}\right)$ & Final $\left(\mathbf{T}_{\mathrm{f}}\right)$ & $\begin{array}{c}\text { Diferencial } \\
\left(\Delta T=T_{f}-T_{i}\right)\end{array}$ & $\begin{array}{c}\text { Requerida } \\
\left({ }^{\circ} \mathrm{C}\right)\end{array}$ & & \\
\hline may-15 & 27,5 & 52,5 & $+25,0$ & 70,0 & 75,0 & 180,0 \\
\hline jun-15 & 27,3 & 50,8 & $+23,5$ & 70,0 & 72,6 & 163,8 \\
\hline jul-15 & 26,0 & 43,1 & $+17,1$ & 70,0 & 61,6 & 123,8 \\
\hline ago-15 & 26,2 & 35,3 & $+9,1$ & 70,0 & 50,4 & 79,1 \\
\hline sep-15 & 27,5 & 51,7 & $+24,2$ & 70,0 & 73,9 & 115,4 \\
\hline oct-15 & 26,4 & 42,9 & $+16,5$ & 70,0 & 61,3 & 119,3 \\
\hline nov-15 & 26,0 & 41,0 & $+15,0$ & 70,0 & 58,6 & 104,6 \\
\hline dic-15 & 30,4 & 54,2 & $+23,8$ & 70,0 & 77,4 & 171,3 \\
\hline ene-16 & 31,6 & 60,4 & $+28,8$ & 70,0 & 86,3 & 197,5 \\
\hline feb-16 & 32,6 & 43,7 & $+11,1$ & 70,0 & 62,4 & 74,5 \\
\hline mar-16 & 28,5 & 52,9 & $+24,4$ & 70,0 & 75,6 & 175,9 \\
\hline Abr-16 & 28,3 & 53,9 & $+25,6$ & 70,0 & 77,0 & 178,9 \\
\hline Media mensual & 28,2 & 48,5 & $+20,3$ & 70,0 & 69,3 & 140,3 \\
\hline
\end{tabular}

El agua presentó una temperatura media de $28,2^{\circ} \mathrm{C}$ a la entrada del sistema solar termosifónico. Durante los meses analizados en el período 2015-2016, se determinó que el agua presentó la temperatura más alta en el mes de abril de $2016\left(33,63{ }^{\circ} \mathrm{C}\right)$. En general, el sistema termosifónico logró un incremento promedio de $20,3{ }^{\circ} \mathrm{C}$, lo que indica que permitió cubrir aproximadamente $69,3 \%$ de la energía necesaria para alcanzar la temperatura del agua requerida para las operaciones de limpieza de la lechería y sanitización de los equipos de ordeño (Figura 5).

El sistema termosifónico, al igual que el fotovoltaico, siguió un comportamiento gaussiano de producción, en donde su máximo rendimiento se alcanzó entre las 11 y $12 \mathrm{~h}$ del día (Figura 6), lo que evidencia una eficiencia de aproximadamente $55,3 \%$.

El sistema solar termosifónico fue capaz de aportar en promedio 140,34 kWh mensuales, lo que equivale a un promedio de 55,25 $\mathrm{kg}$ de $\mathrm{CO}_{2}$ anual (7,62 kg por mes, Cuadro 5).

En la lechería del ITCR-SSC se logró un ahorro de aproximadamente US\$1152 con el empleo de ambos sistemas térmicos solares durante el período analizado. Este ahorro representó el 35,57\% del total (US\$3257,09) de la factura anual de electricidad (Cuadro 6 y 7). 


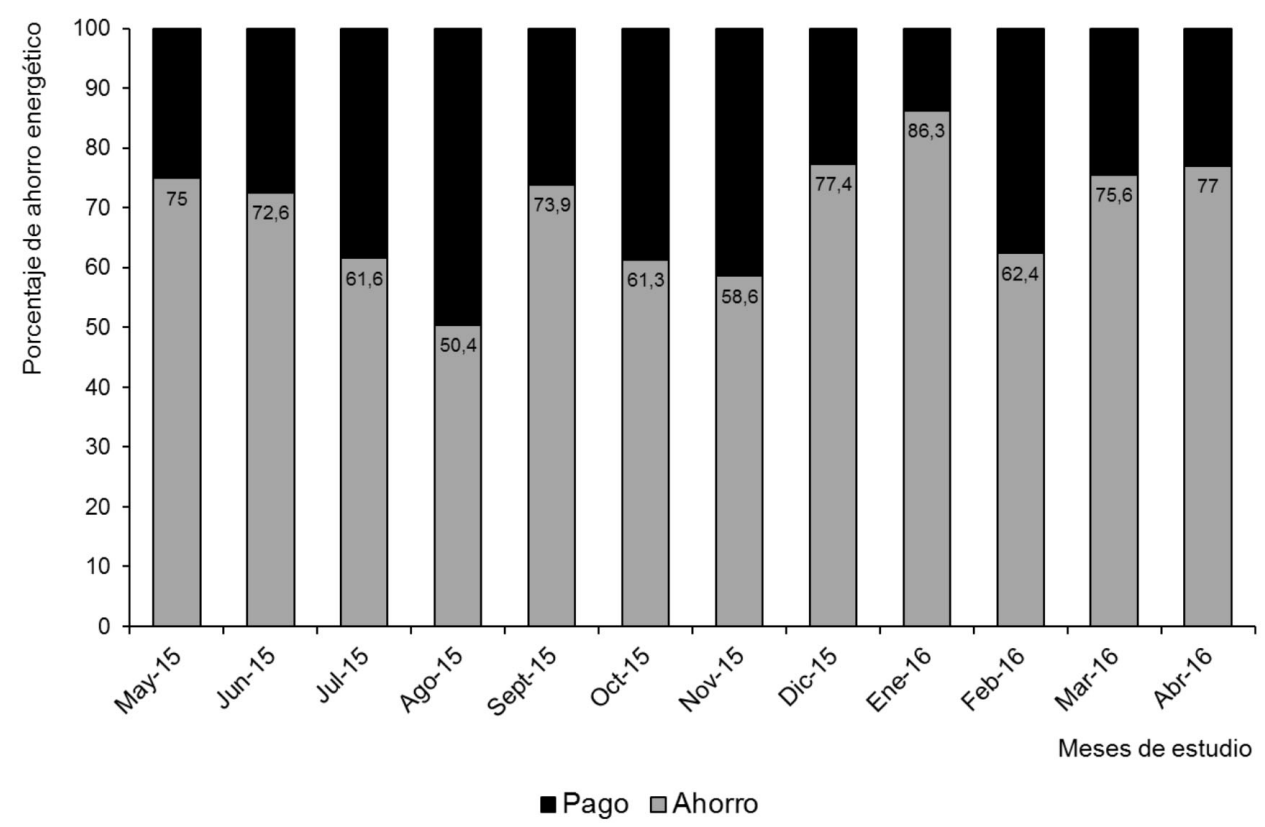

Figura 5. Ahorro energético porcentual logrado con el calentamiento del agua por medio del sistema termosifónico solar, de mayo de 2015 a abril 2016, lechería del Instituto Tecnológico de Costa Rica, Sede Regional San Carlos (ITCR-SSC). Costa Rica.

Figure 5. Percentage of energetic saving, reached by using the thermosyphonic solar system for water heating, from May 2015 to April 2016, Technolgy Institute of Costa Rica's Dairy, San Carlos Headquarter (ITCR-SSC). Costa Rica.

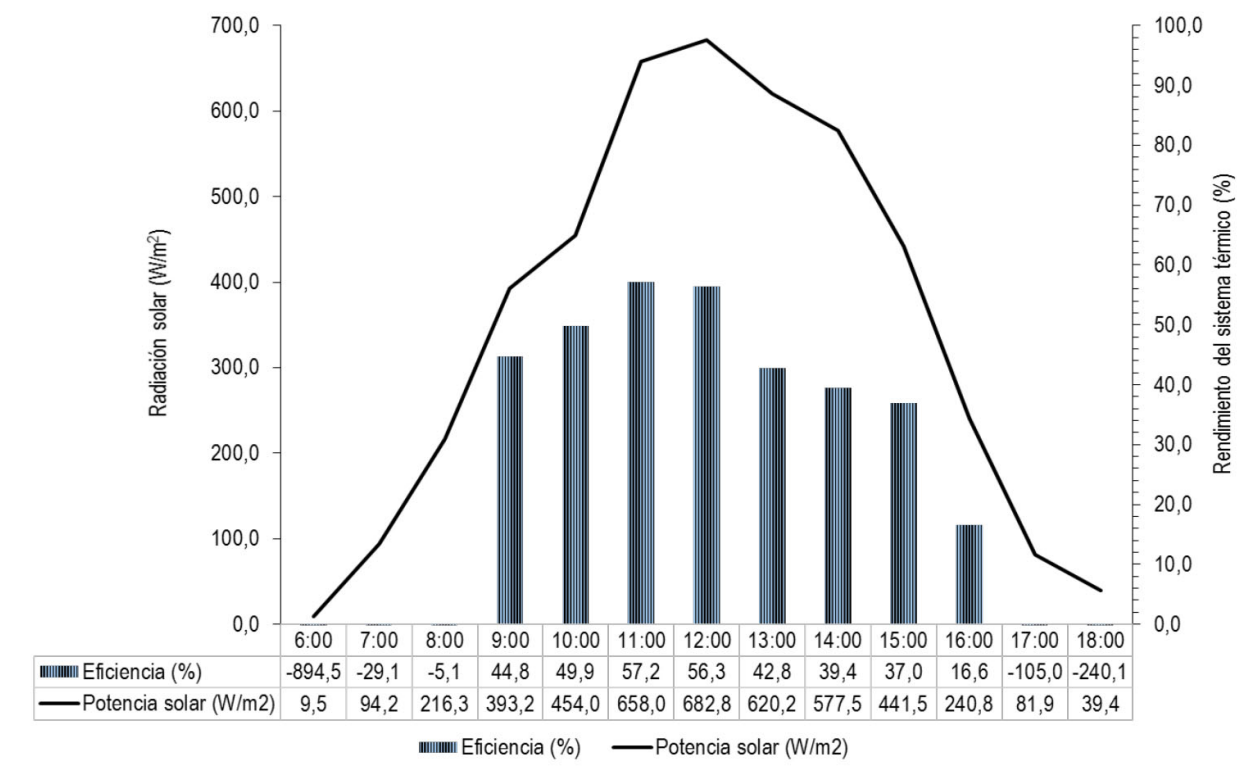

Figura 6. Rendimiento medio anual del sistema termosifónico a lo largo de un día, lechería del Instituto Tecnológico de Costa Rica, Sede Regional San Carlos (ITCR-SSC). Costa Rica.

Figure 6. Annual efficiency mean of the thermosyphonic system along a day, in the Technolgy Institute of Costa Rica's Dairy, San Carlos Headquarter (ITCR-SSC). 
Cuadro 5. Kilogramos de $\mathrm{CO}_{2}$ equivalente a la cantidad de energía generada por los colectores térmicos instalados en la lechería del Instituto Tecnológico de Costa Rica, Sede Regional San Carlos (ITCR-SSC) si procedieran de la electricidad. Calculado de Mayo 2015 a Abril 2016. Costa Rica.

Table 5. Kilograms of $\mathrm{CO}_{2}$ equivalent to the energy generated by the thermal collectors installed at the dairy of the Technology Institute of Costa Rica, San Carlos Headquarter (ITCR-SSC) if they would proceed from electricity. Calculate from May 2015 to April 2016. Costa Rica.

\begin{tabular}{|c|c|c|c|c|c|}
\hline \multirow[t]{2}{*}{ Mes } & \multicolumn{2}{|c|}{ Temperatura del agua $\left({ }^{\circ} \mathrm{C}\right)$} & \multirow{2}{*}{$\begin{array}{c}\text { Energía diaria } \\
\text { aportada (kJ/día) }\end{array}$} & \multirow{2}{*}{$\begin{array}{c}\text { Energía mensual } \\
\text { aportada }(\mathrm{kWh} / \mathrm{mes})\end{array}$} & \multirow{2}{*}{$\begin{array}{r}\text { Kg de } \mathrm{CO}_{2} \\
\text { equivalentes }\end{array}$} \\
\hline & Inicial $\left(T_{i}\right)$ & Final $\left(T_{f}\right)$ & & & \\
\hline Mayo 15 & 27,5 & 52,5 & 20900,1 & 180,0 & 10,0 \\
\hline Junio 15 & 27,3 & 50,8 & 19650,4 & 163,8 & 9,1 \\
\hline Julio 15 & 26,0 & 43,1 & 14379,4 & 123,8 & 6,9 \\
\hline Agosto 15 & 26,2 & 35,3 & 7600,7 & 79,1 & 4,4 \\
\hline Septiembre 15 & 27,5 & 51,7 & 20273,2 & 115,5 & 6,4 \\
\hline Octubre 15 & 26,4 & 42,9 & 13857,6 & 119,3 & 6,6 \\
\hline Noviembre 15 & 26,0 & 41,0 & 12552,8 & 104,6 & 5,8 \\
\hline Diciembre 15 & 30,4 & 54,2 & 19891,9 & 171,3 & 9,5 \\
\hline Enero 16 & 31,6 & 60,4 & 22940,6 & 197,5 & 11,0 \\
\hline Febrero 16 & 32,6 & 43,7 & 9248,5 & 74,5 & 4,2 \\
\hline Marzo 16 & 28,5 & 52,9 & 20422,1 & 175,9 & 9,8 \\
\hline Abril 16 & 28,3 & 53,9 & 21473,2 & 178,9 & 10,0 \\
\hline Media mensual & 28,2 & 48,5 & 16932,5 & 140,3 & 7,8 \\
\hline
\end{tabular}

Cuadro 6. Ahorro mensual y anual (en dólares estadounidenses) por la producción energética generada por los sistemas fotovoltaicos y termosifónicos y, porcentaje representativo respecto al gasto en la lechería del Instituto Tecnológico de Costa Rica, Sede Regional San Carlos (ITCR-SSC). Costa Rica. Mayo 2015 a abril 2016.

Table 6. Monthly and annual savings (in American dollars) for energy production by the photovoltaic and the hermosyphonic systems, and the representative percentage of the expenses at the dairy of the Technology Institute of Costa Rica, San Carlos Headquarter (ITCR-SSC). Costa Rica. May 2015 to April 2016.

\begin{tabular}{|c|c|c|c|c|c|}
\hline Mes & $\begin{array}{c}\text { Energía total } \\
\text { producida }(\mathbf{k W h})\end{array}$ & $\begin{array}{c}\text { Costo por energía } \\
(\$)\end{array}$ & $\begin{array}{c}\text { Costo por alumbrado } \\
\text { público (\$) }\end{array}$ & $\begin{array}{c}\text { Impuesto de } \\
\text { ventas }(\$)\end{array}$ & Ahorro total (\$) \\
\hline Mayo 15 & 359,0 & 91,7 & 2,5 & 4,6 & 98,7 \\
\hline Junio 15 & 325,0 & 87,2 & 2,2 & 4,4 & 93,8 \\
\hline Julio 15 & 308,0 & 84,9 & 2,1 & 4,2 & 91,2 \\
\hline Agosto 15 & 280,0 & 81,2 & 1,9 & 4,1 & 87,2 \\
\hline Septiembre 15 & 289,0 & 82,4 & 2,0 & 4,1 & 88,5 \\
\hline Octubre 15 & 307,0 & 84,7 & 2,1 & 4,2 & 91,1 \\
\hline Noviembre 15 & 301,0 & 84,0 & 2,1 & 4,2 & 90,2 \\
\hline Diciembre 15 & 383,0 & 94,9 & 2,6 & 4,7 & 102,2 \\
\hline Enero 16 & 420,0 & 99,8 & 2,9 & 5,0 & 107,7 \\
\hline Febrero 16 & 289,0 & 82,3 & 2,0 & 4,1 & 88,4 \\
\hline Marzo 16 & 442,0 & 102,6 & 3,0 & 5,1 & 110,8 \\
\hline Abril 16 & 427,0 & 100,7 & 2,9 & 5,0 & 108,7 \\
\hline Total & 4128,0 & 1076,3 & 28,5 & 53,8 & 1158,5 \\
\hline
\end{tabular}


Cuadro 7. Ahorro económico porcentual derivado de la producción energética generada por los sistemas fotovoltaicos y termosifónicos, instalados en la lechería del Instituto Tecnológico de Costa Rica, Sede Regional San Carlos (ITCR-SSC). Costa Rica. Mayo 2015 a abril 2016.

Table 7. Percentage of economic saving derived from the energy production generated by the photovoltaic and thermosyphonis systems installed at the dairy of the Technology Institute of Costa Rica, San Carlos Headquarter (ITCR-SSC). Costa Rica. May 2015 to April 2016.

\begin{tabular}{lc}
\hline Concepto & Monto (US\$) \\
\hline Factura eléctrica sin instalación de equipo solar & 3257,1 \\
Factura eléctrica con instalación de equipo solar & 2098,5 \\
Ahorro & 1158,5 \\
Porcentaje de ahorro (\%) & $\mathbf{3 5 , 6}$ \\
\hline
\end{tabular}

En el año cero se haría el pago de la inversión del proyecto (US\$10.000), y cada año que pasa esta inversión se pagaría con el ahorro que se generaría en la factura eléctrica (US\$1.158,5), por lo que, en el año 8 se lograría el retorno de la inversión, y a partir de ahí el productor recibiría los beneficios netos de la inversión, ya que los ahorros se seguirían recibiendo por el periodo de vida útil de los equipos (más de 25 años).

\section{Discusión}

Los sistemas solares instalados en la lechería del ITCR-SSC lograron generar un promedio mensual de aproximadamente $344 \mathrm{kWh}$ y un total de $4130 \mathrm{kWh}$, equivalentes a US\$1.160 capaz de cubrir el 35\% del consumo eléctrico total anual (US\$3.260). De ese consumo eléctrico, una parte era destinada a calentar el agua para el lavado de equipos y desinfección, procesos que actualmente se cubren en un $74 \%$ por calentamiento térmico, a través de los sistemas termosifónicos. Además, el agua tiene un alto poder calorífico $\left(4187 \mathrm{~J} / \mathrm{kg} \cdot{ }^{\circ} \mathrm{C}\right.$ ) (Poling et al., 1987) y una vez caliente se deposita en un tanque sellado, posteriormente, se puede disponer siempre de ésta para las operaciones de limpieza y sanitización que se realizan en la lechería, para asegurar la inocuidad en esta unidad productiva.

Se puede afirmar que la cantidad de energía producida depende en alto grado de la intensidad de la radiación solar incidente, independientemente de la temperatura, la nubosidad y las precipitaciones de la zona, las cuales son constantes y abundantes a lo largo de todo el año.

La relación entre la cantidad de radiación solar incidente y la energía generada a lo largo del día, revela una alta correlación, razón por la cual siempre es preciso colocar los sistemas en la posición y ángulo que aproveche al máximo la luz incidente (Benítez-Salazar et al., 2013). Además, el rendimiento de los sistemas térmicos solares instalados en el ITCR-SSC es consecuencia del tipo de material con el que están construidos (González, 2009). En este estudio se determinó que el rendimiento disminuyó conforme aumentó la diferencia de temperaturas con respecto de la irradiación incidente (a la salida y puesta del sol, y durante la noche). Al mediodía, tanto la irradiación como la temperatura ambiente tienen poca variación, pues es mayor la temperatura de entrada de agua al colector y más calor transfieren estos hacia el medio exterior (Joshi et al., 2005); es decir, de la radiación que llega a los colectores, se aprovecha más al mediodía porque el propio colector pierde menos energía que cuando no hay radiación.

De acuerdo con el ICE (2010), el 76\% de la energía generada en Costa Rica es de origen hidroeléctrico; $12 \%$ de origen geotérmico, $4 \%$ de origen eólico y $1 \%$ de biomasa. Solamente un $7 \%$ del total producido se genera con fuente térmica, por lo que, el $93 \%$ de la energía generada en el país procede de fuentes no fósiles, lo que hace que 
la cantidad de $\mathrm{CO}_{2}$ que se evita emitir a la atmósfera con los sistemas solares no sea tan significativo como puede serlo en otros países más dependientes de los combustibles fósiles.

Aunque las centrales hidroeléctricas no contaminen en términos de 0 emisión de gases de efecto invernadero (GEI), no significa que no suponga un impacto ambiental sobre las regiones donde se construyen, como los desvíos de los ríos e impacto directo sobre el microclima y fauna piscícola, deforestación y expropiación de tierras por inundación. Debido a esto, la energía solar destaca por ser una tecnología sostenible, viable y factible en diversos usos domésticos, industriales y en aplicaciones tecnológicas (Iglesias-Ferrer y Morales-Salas, 2013).

Es preciso señalar que, en el monto calculado, no se tiene en cuenta el costo ambiental que supone la implantación de los sistemas solares, el cual elevaría significativamente el ahorro de la factura eléctrica, ya que actualmente las empresas no contemplan los costos ambientales que generan sus actividades. Las nuevas normativas ambientales y la presión de las organizaciones no gubernamentales y de los organismos de crédito internacionales, están modificando esta actitud y han llevado a las empresas a prestar cada vez más atención a esos costos.

Con el tiempo, la sociedad adoptará las energías renovables, ya que las reservas de combustibles fósiles son limitadas y sólo son generadas con el transcurso de tiempos geológicos. Por lo tanto, la pregunta no es si la sociedad adoptará las energías renovables, sino cuándo lo hará. Los tiempos de vida de los combustibles fósiles podrían ampliarse gracias a las nuevas tecnologías de extracción, pero la necesidad de minimizar los efectos nocivos del cambio climático es un problema más inmediato que el agotamiento de los combustibles fósiles (Timmons et al., 2014).

\section{Conclusiones}

Los sistemas de energía solar son una alternativa para disminuir la dependencia de los combustibles fósiles de las explotaciones productivas agropecuarias de la Región Huetar Norte de Costa Rica. En este trabajo se demostró que la Región Huetar Norte es una zona donde estos sistemas han contribuido a mejorar no sólo la eficiencia de estas explotaciones, sino también las operaciones que en ellas se realizan. Además, el uso de estos sistemas para la generación de energías limpias contribuye a disminuir la emisión de gases de efecto invernadero y, por ende, la huella de carbono de estas explotaciones; lo que implica una diferenciación comercial de la producción de quienes los usen, por medio de un sello ecológico o sello verde.

\section{Literatura citada}

ASHRAE (American Society of Heating, Refrigerating and Air Conditioning Engineers). 2003. Standard ANSI/ASHRAE 932003: Methods of testing to determine the thermal performance of solar collectors. ASHRAE, USA.

Benítez-Salazar, V.H., G.A. Torres-Valverde, L.A. Gámez-Valdéz, y J.H. Pacheco-Ramírez. 2013. Sistema fotovoltaico de iluminación solar. Epistemus 15:86-92.

Díaz, J.F. 2015. Conceptos de energía solar térmica. Blog eficiencia energética y utopía. https://juanfrancisco207.wordpress. com/2015/01/31/conceptos-de-energia-solar-termica/ (consultado 15 jul. 2016).

Espejo-Marín, C. 2004. La energía solar fotovoltaica en España. Nimbus 13-14:5-31.

González, J. 2009. Energías renovables. Editorial Reverté SA, ESP.

Henley, E.J. 1973. Sistemas de unidades. En: E.J. Henley, y E.M. Rosen, editores, Cálculo de balances de materia y energía Editorial Reverté SA, ESP. p. 31-34. 
ICE (Instituto Costarricense de Electricidad). 2015. Costa Rica: matriz eléctrica. gobierno.cr/wp-content/uploads/2015/06/ matriz_folleto_ICE.pdf (consultado 8 jul. 2016).

Iglesias-Ferrer, J.M., y J. Morales-Salas. 2013. Dimensionado de un sistema térmico solar mediante simulación y su validación energética. Ingeniería Energética 34:55-65.

IMN (Instituto Meteorológico Nacional). 2016. Factores de emisión gases de efecto invernadero. 6a ed. IMN, CRC. http:// cglobal.imn.ac.cr/documentos/factores-de-emision-gei-sexta-edicion (consultado 30 ene. 2017).

Joshi, S.V., R.S. Bokil, and J.K. Nayak. 2005. Test standards for thermosyphon-type solar domestic hot water system: review and experimental evaluation. Solar Energy 78:781-798.

Khan, M., A. Abdul-Malek, M. Mithu, and D. Das. 2010. Design, fabrication and performance evaluation of natural circulation rectangular box-type solar domestic water system. IJRER 2:164-177.

Lamela, A. 2005. La sostenibilidad, un reto global ineludible. Informes de la Construcción 57:499-500.

Matthews, C. 2006. La ganadería amenaza el medio ambiente. Organización de las naciones Unidas para la Agricultura y la Alimentación. http://www.fao.org/Newsroom/es/news/2006/1000448/index.html (consultado el 29 de jun. 2016).

MINAET (Ministerio de Ambiente, Energía y Telecomunicaciones). 2011. VI Plan Nacional de energía 2012-2013. http://www. dse.go.cr/es/03Publicaciones/01PoliticaEnerg/VI_Plan_Nacional_de_Energia_2012-2030.pdf (consultado 25 may. 2016).

Poling, B.E., J.M. Prausnitz, and R.C. Reid. 1987. The properties of gases and liquids. $4^{\text {th }}$ ed. McGraw-Hill interamericana, ESP.

Prosperi, M., et A. Croci. 2005. Dossier solare térmico. Proyecto RES \& RUE Disemination. http://www.ape.fvg.it/wp-content/ uploads/dlm_uploads/2016/03/ADICONS-Solare-termico.pdf (consulté 15 jul. 2016).

Quirós, L. 2011. Construir América Central y el Caribe. Rev. Construir. http://revistaconstruir.com/construccion-sostenible-unreto-para-la-region/836/ (consultado 28 jun. 2016).

Shyam, S.N. 2005. La energía solar: conceptos básicos y su utilización. Universidad Nacional, Heredia, CRC. http://www. sistemamid.com/panel/uploads/biblioteca/2014-06-27_10-14-37105995.pdf (consultado 28 jun. 2016).

Taiz, L., and E. Zeiger. 2002. Photosynthesis: The light reactions. In: L. Taiz, editor, Plant physiology. $5^{\text {th }}$ ed. Sinauer Associates Inc., MA, USA. p. 163-194.

Timmons, D., J. M. Harris, y B. Roach. 2014. La economía de las energías renovables. Tufts University, MA, USA. http://www. ase.tufts.edu/gdae/education_materials/modules/EconomiaEnergiasRenovables.pdf (consultado 3 oct. 2016). 